Introduction The field of occupational health nursing is poorly developed in Zimbabwe. This is evidenced by lack of trained professionals and grossly limited occupational health nursing services in the country. This is against a background of high work activity in the mining and agricultural sectors that pose significant occupational health and safety risks. Lack of adequate and comprehensive occupational health nursing services compromises the health and safety of most workers in the work environment.

Methods A cross sectional survey was carried out in major mining companies in Zimbabwe. A telephonic survey was carried out amongst ten nurses in these mining companies to ascertain the main challenges faced by the nurses working in these mines. Results All the nurses noted lack of recognition of occupational health qualifications by the nursing council as a major hindrance in the development and practice of occupational health nursing. Lack of educational institutions offering diploma or degree courses in occupational health nursing was highlighted by all the survey participants as one of the major challenges. Eighty per cent of the nurses noted the most doctors manning the mine clinics did not have a qualification in occupational health thereby presenting difficulties in running the occupational health clinics.

Conclusion Capacitation of occupational health nurses with occupational health training in Zimbabwe remains key. In addition, it is of paramount significance for regulatory bodies like the Nurses Council to embrace and recognise the discipline of occupational health nursing. Recognition of occupational health nursing by regulatory bodies in the country has the capacity of stimulating the growth and maturation of the field of occupational health. Technical assistance in this field critically needed in order to develop the occupational health human capital in this field.

\section{A TRIAL FOR IMPROVEMENT OF WORKPLACE PATROL FOR SMALL-SCALE ENTERPRISES BY OCCUPATIONAL HEALTH NURSES, USING SELF-MADE CHECKLIST AND UTILISATION MANUAL}

\begin{abstract}
1,2,3 Yumi Nakao* ${ }^{2,3,4}$ Yuriko Hachiya, ${ }^{3}$ Yukiko Ogawa, ${ }^{3}$ Satomi Kakimori, ${ }^{3}$ Yumiko Iwasa, ${ }^{2}$ Fumiko Ichikawa, ${ }^{2}$ Susumu Oda. 'Work and Health Occupational Health Consultant Office NAKAO, Fukuoka, Japan; ${ }^{2}$ Fukuoka Occupational Health Support Centre, Fukuoka, Japan; ${ }^{3}$ Fukuoka-Central Division of the Fukuoka Occupational Health Centre, Fukuoka, Japan; ${ }^{4}$ School of Health Sciences Occupational and Community Health Nursing, UOEH, Fukuoka, Japan
\end{abstract}

\subsection{6/oemed-2018-ICOHabstracts.1009}

Introduction In Japan, employees are obliged to listen to a doctor's advice when abnormal results are found in periodical medical examinations. Then, Health guidance by occupational health nurses(OHNs) etc. is required as necessary in enterprises with 50 or more workers. However, workers have not received the same services in the small-scale enterprises with less than 50 workers. The Authors will report on workplace patrol using a self-made checklist and its utilisation manual in the small-scale enterprises.

Methods

1. targets: 71 small-scale enterprises with less than 50 workers, Fukuoka, Japan

2. period of survey: 28/6/2016 16/6/2017 3)Tools: checklist of workplace patrol and its utilisation manual.

Results Summary of 71 small-scale enterprises:
1. type of occupation: $61 \%$ tertiary industries $13 \%$ secondary industries

2. Appointment of safety and health promoters: $73 \%$ of enterprises were appointed

3. implementation of workplace patrol: $17.6 \%$ in the first visit, $59.4 \%$ in the second or third visit $(\mathrm{p}<0.001)$.

Discussion Most doctors registered in Regional Occupational Health Centres are supposed to do workplace patrols, but they cannot share the time workplace patrol because they were busy with medical examinations and treatment. So because of that we coordinated 10 OHNs. Experiences of OHNs are variable so we made checklist of workplace patrol and its utilisation manual, moreover we asked engineering industrial health hygienists to patrol with them. In general, OHNs are good at health control but they do not have sufficient knowledge and technique to regulate work and working environment.

Conclusion Although our checklists are fundamental, and common to various types of occupation,they are useful for improvement of workplace patrol by OHNs, accompanied with engineering industrial health hygienists. As our services are provided once a year for free, continued support once a year might make employers and employees aware as to what they should do by themselves.

\section{SENSE OF DIFFICULTY, SKILL RETENTION, AND LEARNING STRATEGIES IN WORKPLACE MENTAL HEALTH ACTIVITIES BY OCCUPATIONAL HEALTH NURSES (2ND REPORT)}

Noriko Nishikido*, Mako Ishikawa, Satoko Shimamoto. Graduate School of Nursing, Tokai University, Kanagawa, Japan

\subsection{6/oemed-2018-ICOHabstracts. 1010}

Purpose To clarify the relationship between skill retention in mental health activities and the learning environment/selfimprovement of occupational health nurses.

Methods As per the 1 st report, data obtained by questionnaire survey was used. Participants answered yes or no to 11 items regarding the learning environment, and to 6 items regarding self-improvement, respectively. We compared scores of skill retention according to the presence or absence of a learning environment and self-improvement for different levels of experience by U-test.

Results Through all levels of years of experience, approximately $70 \%-80 \%$ of nurses received advice regarding mental health activities in the workplace, approximately $80 \%-90 \%$ of them set tasks and targets on their own and performed selfevaluations. On the other hand, approximately 30\% of nurses received support such as from university. Furthermore, while the rate of individuals with experience in performing studies was approximately $20 \%$ among novices, the rate tended to increase with years of experience. Novices had opportunities to consult occupational health nurses of other companies, and subscribed to relevant magazines, which significantly correlated with the sense of skill retention. Among mid-career nurses, having experience in performing studies significantly correlated with this. In the management period, a significant correlation was found with having case study groups and study groups at the workplace, and in the late-management period, increased sense of skill retention was observed in the group that set tasks and targets and performed evaluations on their own. 
Discussion We found that the learning environment and selfimprovement in terms of the sense of skill retention regarding mental health activities differed according to years of experience, and it appeared that at each career stage, there are characteristics in the learning environment/self-improvement required to improve the sense of skill retention.

\section{SENSE OF DIFFICULTY, SKILL RETENTION, AND LEARNING STRATEGIES IN WORKPLACE MENTAL HEALTH ACTIVITIES BY OCCUPATIONAL HEALTH NURSES (1ST REPORT)}

Noriko Nishikido*, Mako Ishikawa, Satoko Shimamoto. Graduate School of Nursing, Tokai University, Kanagawa, Japan

\subsection{6/oemed-2018-ICOHabstracts. 1011}

Purpose To clarify the sense of difficulty in mental health activities by occupational health nurses, as well as the characteristics of skills retention and future learning tasks.

Methods Self-administered questionnaire surveys were conducted. A cluster analysis was performed of 36 items regarding mental health activities, which were classified into categories. The years of experience were divided into 5 levels, i.e. 0-4 (novice), 5-9 (early-mid), 10-14 (late-mid), 15-19 (pre-management), and 20 or more years (late management), then the responses were compared between the 5 levels.

Results Mental health activities were classified into 6 categories, including the 'construction of relationships of trust between workers and the manager, and data collection', 'assessment and support of individual consultations', 'support for job reinstatement and cooperation with relevant individuals within and outside of the enterprise', 'provision of information to workplace groups and organisations', 'construction of a mental health support system as a workplace organisation', and 'support for cases that are difficult to manage'. As the years of experienced increased, the number of nurses who had difficulty in their activities decreased. However, even in the management level, there was a strong sense of difficulty in 'support for cases that are difficult to manage'. The sense of retaining skills increased with the increase in experience. With regards to future learning tasks, for all levels of experience many nurses indicated 'support for cases that are difficult to manage'. Novices indicated 'assessment and support of individual consultations'.

Discussion Suggestions for training measures according to each career level were obtained. Among novices, training is needed for the 'assessment and support of individual consultations', while for nurses in their early- to mid-career or after, 'support for cases that are difficult to manage' as well as focusing on group and organisational support was considered appropriate.
571

CONSTRUCTION OF OCCUPATIONAL HEALTH NURSING DIAGNOSIS SYSTEM FOR GROUP AND ORGANISATION IN MENTAL HEALTH IN JAPAN

${ }^{1} \mathrm{Y}$ Yamaguchi*, ${ }^{2} \mathrm{M}$ Ito, ${ }^{3} \mathrm{~F}$ Akimoto, ${ }^{3} \mathrm{~K}$ Hara, ${ }^{3} \mathrm{~K}$ Masuzawa, ${ }^{4} \mathrm{M}$ Ito, ${ }^{4} \mathrm{H}$ Nakamur, ${ }^{4} \mathrm{M}$ Yanai, Murakami ${ }^{5},{ }^{6} \mathrm{~A}$ Nagano, ${ }^{7} \mathrm{M}$ Seto, ${ }^{8} \mathrm{R}$ Yoshida, ${ }^{9} \mathrm{~K}$ Kono, ${ }^{10} \mathrm{~A}$ Chiba, ${ }^{11} \mathrm{~N}$ Ochiai, ${ }^{12} \mathrm{M}$ Tachikawa, ${ }^{13} \mathrm{~K}$ Inomata. ${ }^{1}$ Sophia University, Tokyo, Japan; ${ }^{2}$ Tokyo Health Care University, Tokyo, Japan; ${ }^{3}$ Nippon Telegraph and Telephone East Corporation, Tokyo, Japan; ${ }^{4}$ Occupational Health Nursing Systematisation Consideration Committee in JAOHN, Fukuoka, Japan Tokyo; ${ }^{5}$ Metro Co., Ltd., Tokyo, Japan; ${ }^{6}$ Suzuki Motor Corporation, Shizuoka, Japan; ${ }^{7}$ LOIN Corporation Health Support Office (Hirai), Tokyo, Japan; ${ }^{8}$ Epson Sales Japan Corp., Tokyo, Japan; ${ }^{9}$ Research Centre for Occupational Health Nursing, Yokkaichi Nursing and Medical Care University, Mie, Japan; ${ }^{10}$ Aomori University of Health and Welfare, Aomori, Japan; ${ }^{11}$ The University of Shimane, Shimane, Japan; ${ }^{12}$ Ube Frontier University, Yamaguchi. Japan; ${ }^{13}$ Teikyo Heisei University, Tokyo, Japan

\subsection{6/oemed-2018-ICOHabstracts. 1012}

Introduction In order to conduct effective occupational health nursing $(\mathrm{OHN})$ services to groups and organisations, it is necessary to do assessment, planning, implementation and evaluation in line with the nursing process continuously. For that, systematisation of $\mathrm{OHN}$ diagnosis is necessary so that the three processes of planning (nursing diagnosis, nursing goal, nursing care) can be systematically utilised in particular. The purpose of this study is to develop OHN diagnosis (diagnostic index, related factor), nursing goal, nursing care in the mental health for groups and organisations, taking 'chronic high stress state' as an example.

Methods Development was advanced by 7 OHN researchers and $10 \mathrm{OHN}$ practitioners, receiving supervision, referring NANDA-I nursing diagnosis and Arizona University NIC. 'Chronic high stress state' which is one of nursing diagnosis in mental health in OHN, an OHN science system was constructed in a format systematically showing three processes.

Result In the nursing diagnosis name 'chronic high stress state', examples of diagnostic indicators were the bad results in stress survey at workplace and the number of workers on leave due to mental disorders, Examples of relevant factors are the qualitative burden of work, the quantitative burden of work. In addition, the nursing goal showed that mental health disorder decreased, new incidences of mental health disorder decreased, nursing care showed recommendations to managers, recommendations to health and safety committee, and so on.

Discussion By systematically arranging the three processes of 'chronic high stress state', the problems of group and organisation (nursing diagnosis and diagnostic index) and its factors (related factor), targeted form (nursing goal), appropriate nursing support, these relationships became clear. In addition, 'Chronic High Stress Condition' is considered to be the basis for constructing a highly versatile system with high support needs in the development of OHN diagnostic system in mental health. 